Relations industrielles

Industrial Relations

\title{
AFL-CIO : Labor United. By Arthur J. Goldberg. New-York: McGraw-Hill Book Company, Inc., 1956, pp. 309. \$5.00.
}

\section{Roger Chartier}

Volume 12, numéro 4, octobre 1957

URI : https://id.erudit.org/iderudit/1022523ar

DOI : https://doi.org/10.7202/1022523ar

Aller au sommaire du numéro

Éditeur(s)

Département des relations industrielles de l’Université Laval

ISSN

0034-379X (imprimé)

1703-8138 (numérique)

Découvrir la revue

Citer ce compte rendu

Chartier, R. (1957). Compte rendu de [AFL-CIO : Labor United. By Arthur J.

Goldberg. New-York: McGraw-Hill Book Company, Inc., 1956, pp. 309. \$5.00.]

Relations industrielles / Industrial Relations, 12(4), 409-409.

https://doi.org/10.7202/1022523ar

Tous droits réservés (C Département des relations industrielles de l’Université Laval, 1957
Ce document est protégé par la loi sur le droit d'auteur. L’utilisation des services d'Érudit (y compris la reproduction) est assujettie à sa politique d'utilisation que vous pouvez consulter en ligne.

https://apropos.erudit.org/fr/usagers/politique-dutilisation/ 


\section{RECENSIONS - BOOK REVIEWS}

Britain Views Our Industrial Relations. By Mark J. Fitzgerald, c.s.c., Notre Dame, Indiana: University of Notre Dame Press, 1957, pp. 221.

A la suite de la seconde guerre mondiale, plusieurs missions économiques de pays européens sont venues étudier aux Etats-Unis les méthodes de production et le régime des relations du travail. Entre 1948 et 1953, sous les auspices de l'Anglo-American Council, 67 gnoupes comprenant un total de 900 représentants des syndicats et des entreprises de Grande-Bretagne ont ainsi eu la possibilité d'analyser la politique des compagnies amérioaines, le rôle des syndicats ouvriers, les standards de rémunération ainsi que les méthodes de distribution du revenu. Chacun de ces groupes a publié un raport de ses observations. Le présent ouvrage étudie ces rapports, les résume et met en évidence les grandes lignes de leurs conclusions. Ici au Canada, si nous connaissons assez bien le régime des relations du travail chez nos voisins. celui de la GrandeBretagne est souvent inconnu. Parce que cet ouvrage met constamment en parallèle l'un et l'autre, dans son ensemble il permet au lecteur de faire des comparaisons assez intéressantes sur des conceptions et des pratiques de l'organisation de la production par les divers agents. Comme ces observations proviennent d'Anglais de Grande-Bretagne, dont l'expérience de la vie industrielle est très longue, elles ont pour nous une valeur particulière. L'auteur de cette synthèse est le R.P. Mark Fitzgerald, professeur d'économique à l'Université Notre Dame, dont la réputation dans le domaine des relations industrielles est bien connue.

\section{AFL-CIO : Labor United. By Arthur I.}

Goldberg. New-York: McGraw-Hill Book Company, Inc., 1956, pp. 309. $\$ 5.00$.

Voici un volume opportun, et dont la valeur comme instrument de référence sur la fusion des deux grandes centrales syndicales américaines est indiscutable.
L'auteur, aviseur légal de l'AFL-CIO, met naturellement lacent sur les aspects juridiques du nouvel organisme, et c'est dans ce domaine que son travail a le plus d'utilité.

Mais Arthur Goldberg ne s'en tient pas qu'au légalisme. Les quatre premiers chapitres relatent brièvement l'histoire du syndicalisme américain depuis un peu plus d'un demi-siècle: on y découvre les origines de l'AFL, puis du CIO, on asiste à la scission des années trente, puis à la lente progression vers l'unité.

Les trois chapitres suivants s'attachent à l'explication de la nouvelle constitution, décrivant l'arrière-plan, le sens et les effets probables des passages les plus importants.

L'auteur, dans un autre chapitre, esquisse une critique sommaire de la fameuse thèse du «monopole syndical ». I! situe ensuite le rôle de la nouvelle centrale unifiée en face du communisme, de la corruption et de la «discrimination 》 à l'intérieur de ses cadres. Il donne enfin un bref aperçu de la politique économico-sociale de l'AFL-CIO, de ses attitudes sur le plan international et dans le domaine politique. Un dernier chapitre établit une projection du syndicalisme dans lavenir. En appendice. le lecteur trouvera tous les textes officiels importants.

Oeuvre de vulgarisation, qui ne satisfera ni l'historien ni l'économiste, mais qui rendra service sur le plan légal; oeuvre utile, destinée au lecteur moyen; oeuvre d'optimisme et de sympathie syndicale. A lire.

\section{Roger Chartier}

I'influence $d u$ syndicalisme national catholique sur le droit syndical québecois, Jean-Réal Cardin, Montréal: «Les cahiers de l'Institut social populaire », no 1 , juillet 1957, pp. 78. \$1.25. 